\title{
Wohlfahrtsstaat und Gewerkschaftsmacht im Finanzmarkt-Kapitalismus: Der Fall Deutschland
}

\author{
Hans-Jürgen Urban
}

Soziale Sicherungssysteme und Gewerkschaften haben durch ihre Beiträge zur Stabilisierung von Konjunktur und Beschäftigung in der Finanz- und Wirtschaftskrise an Ansehen gewonnen. Dieser Ansehensgewinn sollte jedoch die grundlegenden Veränderungen, die beiden in den letzten Jahren zu schaffen gemacht haben, nicht überdecken. Der Beitrag versucht, die Wechselbeziehungen zwischen der Transformation des deutschen Wohlfahrtsstaates und den gewerkschaftlichen Machtverlusten in der These von der Downsizing-Spirale auf den Begriff zu bringen. ${ }^{1}$

\section{Widersprüchliche Entwicklungen}

Die Zeitdiagnosen verwirren. Ausgerechnet in der schwersten Krise des Nachkriegskapitalismus erleben mit den sozialen Sicherungssystemen und den Gewerkschaften wichtige Basisinstitutionen des Wohlfahrtsstaates einen deutlichen Anerkennungszuwachs. So überraschte der nicht als sozialstaatsfreundlich geltende Sachverständigenrat zur Begutachtung der gesamtwirtschaftlichen Entwicklung, als er mit Blick auf die staatliche Stabilisierung der Wirtschaft eindringlich warnte, dass „Reformen, die auf eine Zurückdrängung der Staatstätigkeit, einen schlankeren Sozialstaat und eine weniger progressive Einkommensteuer zielen, zu geringeren automatischen Stabilisierungswirkungen “ führen (SVR 2008, Ziffer 414). Ähnlich freundlich werden Rolle und Zustand der deutschen Gewerkschaften kommentiert. Umgarnt von den politischen Parteien wird ihnen eine „ungeahnte Renaissance“ bescheinigt (Wirtschaftswoche vom 30.3.2009), und mitunter wird anerkennend konstatiert: „Die Gewerkschaften sind zurück“ (Die Zeit vom 30.4.2009).

Diese Einschätzungen verwundern, wurden doch im wissenschaftlichen wie medialen Diskurs an der Zukunftsfähigkeit der sozialen Sicherungssysteme und Gewerkschaften infolge von Globalisierung, sozialem Strukturwandel und Arbeitsmarktverwerfungen durchaus Zweifel an- gemeldet. Eine genauere Betrachtung lässt deutlich werden: Weder für die Zukunft des deutschen Wohlfahrtsstaates noch für die der Gewerkschaften sind Niedergangs- oder Zukunftsszenarien programmiert. Wohl aber existiert ein Zusammenhang zwischen beiden, der hier hervorgehoben werden soll. Die Liberalisierung wohlfahrtsstaatlicher Sektoren hat im deutschen, konservativkorporatistischen Wohlfahrtsregime die Erosion gewerkschaftlicher Macht deutlich befördert. Dabei spielte der Verlust an gewerkschaftlicher Arbeitsmarktmacht infolge rekommodifizierender Arbeitsmarktreformen eine wichtige Rolle. Verluste an Organisations- und institutioneller Macht traten hinzu. Da diesen Machtverlusten die Transformation des Wohlfahrtsregimes zugrunde liegt, dürfte eine Revitalisierung der Gewerkschaften nicht ohne eine erneuernde Reform des Wohlfahrtsstaates zu haben sein. Das erfordert auch von den Gewerkschaften ein verstärktes sozialpolitisches Engagement.

Diese Einschätzung kann auf der Grundlage eines machtressourcen-theoretischen Analyseansatzes entwickelt und unter Rückgriff auf empirische Fallstudien und qualitativ-inhaltliche Politikfeldanalysen plausibel gemacht werden. Dabei sollen weder die Transformation des Wohlfahrtsstaates noch die Einflussverluste der Gewerkschaften monokausal oder erschöpfend erklärt werden; beide müssen - wie der aktuelle Forschungsstand zeigt als multifaktorielle Entwicklungen begriffen werden, in denen sozio-ökonomische, institutionelle und parteipolitische Faktoren ineinandergreifen. ${ }^{2}$ Vielmehr dient die Hervorhebung der Schlüsselrolle der Verteilung von Macht und der Interdependenz zwischen wohlfahrtsstaatlicher Regulierung und gewerkschaftlicher Durchsetzungsfähigkeit der Rückbesinnung auf Zusammenhänge, die in der deutschen Debatte vergessen zu sein scheinen und von deren Wiederentdeckung wertvolle Hinweise für die wissenschaftliche wie gewerkschaftsstrategische Debatte ausgehen könnten.

\section{Machtressourcen-Ansatz als theoretischer Bezugs- rahmen}

Das Geflecht wechselseitiger Beziehungen zwischen wohlfahrtsstaatlicher und gewerkschaftlicher Entwicklung kann

1 Für wertvolle Hinweise danke ich Heiner Dribbusch und Christoph Ehlscheid.

2 Zur aktuellen Wohlfahrtsstaatsdebatte vgl. Obinger/Wagschal 2000a; Lütz/Czada 2004; Obinger/Starke 2007; zur aktuellen Gewerkschaftsforschung vgl. Frege/Kelly 2004; Huzzard et al. 2004; Brinkmann et al. 2008.

\footnotetext{
Hans-Jürgen Urban, Dr. phil., Geschäftsführendes Vorstandsmitglied der IG Metall, zuständig für Sozialpolitik, Gesundheitsschutz und Arbeitsgestaltung. Weitere Arbeitsschwerpunkte: Sozialstaatsforschung, Gewerkschaftsforschung, Gesundheitspolitik, Arbeitspolitik. e-mail: Hans-Juergen.Urban@igmetall.de
} 
durch einen erweiterten MachtressourcenAnsatz erfasst werden, der in der internationalen Wohlfahrtsstaatsforschung der 1980er Jahre und der neueren Gewerkschaftsforschung entwickelt wurde.

\subsection{MACHTRESSOURCEN UND WOHLFAHRTSSTAATEN}

Der Machtressourcen-Ansatz („Power Ressources Theory") wurde in der international vergleichenden Staatstätigkeitsforschung in den 1980er Jahren entwickelt (Korpi 1983; Esping-Andersen 1985; O'Connor/Olson 1998). In dieser mittlerweile einschlägigen „Theorieschule“ (aktuell dazu: Höpner 2009) werden Entstehung und Entwicklung der Wohlfahrtsstaaten in den westlichen Demokratien als Resultate langfristiger Verteilungskonflikte zwischen sozio-ökonomischen Interessengruppen (vor allem Klassen) und von Entscheidungen klassenorientierter Parteienpolitik („partisan politics“) begriffen. Dabei prägt die Ausstattung mit Macht - also mit der Fähigkeit, andere Akteure zu belohnen oder zu bestrafen (Korpi/ Palme 2003b, S. 427) - Handlungspräferenzen und Konfliktstrategien der rational handelnden und mit widersprüchlichen Interessen ausgestatteten Akteure. Die Machtausstattung der Akteure entscheidet letztlich auch über die Ausgestaltung von wohlfahrtsstaatlichen Institutionen, die dabei helfen, „Ressourcen ökonomisch einzusetzen und die Austausch- und Konfliktaustragungsmuster zu stabilisieren" (ebd.) und manifeste Verteilungskonfliktsituationen in stabilere Verteilungsstrukturen zu überführen. Dadurch können Unsicherheiten und Kosten der Mobilisierung, des Erhalts wie der Anwendung (,application“) von Machtressourcen reduziert und Verteilungskonflikte routinisiert werden. Institutionen gelten somit als Resultate vergangener und zugleich als Arenen gegenwärtiger Konflikte, die die sichtbare Spitze eines „Eisberges der Macht“ (Korpi 1998) darstellen, unter der sich der tragende Kiel aus temporär fixierten Kräfteverhältnissen befindet.

Von besonderer Bedeutung im Machtressourcen-Ansatz sind Verteilungskonflikte und Institutionen zwischen Interessenorganisationen von Kapital und Arbeit, deren Interaktionsbeziehungen durch „Austausch wie durch Konflikt gekennzeichnet" sind und deren Verteilungsergebnisse dazu tendieren, „die relative
Machtposition der Teilnehmenden“, aber auch die Gestalt der „Kontext-Faktoren, wie Kapazitäten des Staates, institutionelle Strukturen, ökonomische Entwicklung und die internationale Situation widerzuspiegeln." (Korpi/Palme 2003a, S. 225f.) Da die Verfügung des einzelnen Arbeitnehmers über seine eigene Arbeitskraft aufgrund seiner sozio-ökonomischen Stellung eng begrenzt ist, ist er dem Arbeitgeber unterlegen und wird für ihn „kollektives Handeln zur rationalen Alternative" gegenüber individuellen Handlungen und Kalkülen (ebd., S. 225). Dabei erweist sich auch die Verlängerung des Verteilungskonfliktes in die Arenen der demokratischen Politik als eine rationale Handlungsstrategie, um dort die Machtressource des demokratischen Stimmrechts zur Geltung zu bringen. Aus dieser „Logik kollektiven Handelns" entstanden klassenorientierte Arbeiterparteien als Form der Organisationsmacht organisierter Arbeit.

\subsection{MACHTRESSOURCEN UND GEWERKSCHAFTEN}

In der neueren Gewerkschaftsforschung gewinnt eine Variante des Machtressourcen-Ansatzes an Bedeutung, die auf Erik O. Wright (2000) zurückgeht. Dabei wird Macht als Kapazität von Individuen und Organisationen verstanden, Klasseninteressen zu realisieren, wobei diese Kapazität auch die Fähigkeit einschließt, den Interessen der anderen Klasse entgegentreten zu können. Auf der Grundlage dieser Begriffsbestimmung wird zwischen struktureller („structural power“) und Organisationsmacht (,associational power") der organisierten Arbeit unterschieden. Erstere entsteht ,einfach aus dem Ort des Arbeiters innerhalb des ökonomischen Systems" (Wright 2000, S. 962). Dies gilt zum einen für die Stellung auf dem Arbeitsmarkt, weshalb sich hier von Arbeitsmarktmacht sprechen ließe; und es gilt für die Stellung innerhalb des Produktionsprozesses, was die Bezeichnung als Produktionsmacht nahelegt. Organisationsmacht hingegen entsteht aus der Formierung der Lohnabhängigen zu kollektiven Organisationen. Dabei finden Verteilungskonflikte und Kompromissbildungen in den institutionellen Sphären der Produktion (Unternehmen), des Tausches (Markt) und der Politik (Staat) statt. Betriebsräte („works councils“) werden als die charakteristischen Organisationen in der Sphäre der
Produktion, Gewerkschaften als solche in der Sphäre des Tausches und politische Parteien in der Sphäre der Politik bewertet (Wright 2000, S. 964f.).

In der wissenschaftlichen Debatte ist mit der institutionellen Macht eine weitere Quelle und Dimension von Gewerkschaftsmacht eingebracht worden. Diese entsteht durch die Stellung von Gewerkschaften innerhalb von institutionellen Arrangements des Wohlfahrtsstaates, die aus vorangegangen Konflikten und Aushandlungsprozessen hervorgegangen sind (Dörre 2010). Über historische Platzierung und Verbleib der Gewerkschaften in diesen Institutionen entscheiden historisch gegebene Kräfteverhältnisse, die über kurzzeitige zyklische Schwankungen hinweg stabilisiert werden. Als vierter Quelle gewerkschaftlicher Durchsetzungsfähigkeit wäre von kommunikativer Macht als einer gewerkschaftlichen „soft power“ zu sprechen. Diese kommt in der Fähigkeit zum Ausdruck, erfolgreich in öffentliche Debatten bzw. historisch gegebene hegemoniale Grundstrukturen von Öffentlichkeiten intervenieren zu können (Haug 2009). Der Kampf um die Verteilung kommunikativer Macht entscheidet sich nicht zuletzt im Konflikt um „Meinungsführerschaften“ und anhand der Fähigkeit, in hegemonial vorstrukturierten Öffentlichkeiten eigene Interessenlagen und Verteilungsansprüche platzieren und dafür gesellschaftliche Anerkennung erzeugen zu können.

Auf der Grundlage der Skizze des wohlfahrts- und des gewerkschaftstheoretischen Machtressourcen-Ansatzes lassen sich nun die Wechselbeziehungen zwischen wohlfahrtsstaatlicher Regulierung und gewerkschaftlicher Machtentwicklung genauer fassen. Da Leistungsniveau und institutionelle Ausprägung des Wohlfahrtsstaates maßgeblich durch Verteilungskonflikte und damit durch die relative Machtposition der Gewerkschaften geprägt werden, und da andererseits die Regulierung des Arbeitsmarktes und die wohlfahrtsstaatlichen Institutionen maßgeblich über die strukturelle, institutionelle und Organisationsmacht der Gewerkschaften entscheiden, gewinnt die eingangs formulierte Vermutung über den Zusammenhang zwischen der Liberalisierung des Wohlfahrtsstaates und gewerkschaftlichen Machtverlusten theoretisch an Plausibilität. Dies soll im Folgenden unter Rückgriff auf empirische Fall- und qualitative Politikfeldstudien diskutiert werden. 


\section{Liberalisierung des Wohl- fahrtsstaates und Erosion von Gewerkschaftsmacht}

\subsection{DIE NEUE POLITIK DES WOHL- FAHRTSSTAATES}

Eine machtressourcen-theoretisch inspirierte Analyse des Wechselverhältnisses von wohlfahrtsstaatlicher und gewerkschaftlicher Entwicklung steht für Deutschland noch aus. In der international vergleichenden Wohlfahrtsstaatsforschung besteht jedoch ein weitgehender Konsens dahingehend, dass Kontexte wie Muster wohlfahrtsstaatlicher Entwicklung etwa seit den 1980er Jahren einem tief greifenden Wandel unterliegen. Dabei folgte der Periode wohlfahrtsstaatlicher Expansion in der Prosperitäts-Ära des Nachkriegskapitalismus eine Phase der Beschneidung und des Rückbaus des Wohlfahrtsstaates („retrenchment“) (Pierson 1996, 2001; Obinger/Wagschal 2000a; Lütz/Czada 2004). Obwohl Reichweite und institutionelle Pfade der „New Politics of the Welfare State" innerhalb der liberalen, der sozialdemokratischen und der konservativen Wohlfahrts-Regime differieren, können mit der Re-Kommodifizierung der Arbeitkraft, der generellen „Ausgabendämpfung " für sozialstaatliche Leistungen und der Rationalisierung wohlfahrtsstaatlicher Programme und Institutionen („recalibration“) drei gemeinsame Dimensionen der Restrukturierung der Wohlfahrtsstaaten ausgemacht werden (Pierson 2001). ${ }^{3}$

Auch für die Entwicklung in Deutschland lassen sich empirische Indizien ausmachen, die für einen Zusammenhang zwischen der Verschiebung gesellschaftlicher Kräfteverhältnisse und dem Umund Rückbau des konservativ-korporatistischen Wohlfahrtsstaates sprechen (Obinger/Wagschal 2000b; Lütz/Czada 2004). Dabei stellt seine Transformation in den aktivierenden Staat eine Facette des Übergangs zu einer neuen Kapitalismusformation dar, die als Wandel vom nationalstaatlich organisierten Sozialstaats-Kapitalismus zum transnationalen Finanzmarkt-Kapitalismus beschrieben wurde (Boyer 2000; Windolf 2005). Dieser Übergang geht mit einem tief greifenden Wandel von Sozialpolitik einher. Ihre „Begründung ist eine ausschließlich funktionalistische: Wie viel und welche Sozialpolitik nötig ist, bemisst sich allein nach technischen Erfordernissen der Marktunterstützung und nicht nach normativen Maßstäben von Verteilungsgerechtigkeit oder der Verwirklichung sozialer Bürgerrechte" (Streeck 2009, S. 25; Herv. i. O.). Sozialpolitik wird so Teil einer Liberalisierungspolitik, die „als politisch herbeigeführte und politisch legitimierte Delegation von Allokations- und Distributionsentscheidungen an Märkte" auf die politisch vorangetriebene Durchsetzung von Marktprinzipien in sozialpolitischen Feldern setzt (Höpner et al. 2009, S. 5f.).

Betrachtet man die Entwicklung des deutschen Arbeitsmarktregimes, so wurde dieses durch ein Set aus regulatorischen und distributiven Liberalisierungsmaßnahmen, die vor allem mit den sogenannten Hartz-Reformen einhergingen, grundlegend verändert. Diese beinhalteten vor allem:

- eine Reduzierung der Höhe der Lohnersatzleistungen und eine Verschärfung der Anspruchsvoraussetzungen für ihren Bezug;

- die drastische Verschärfung der Zumutbarkeitsregelungen für Arbeitsuchende; - einen Umbau der Institutionen der Arbeitsverwaltung mit einer deutlichen Einschränkung der Kompetenzen der sozialen Selbstverwaltung.

In der deutschen Sozialstaatstradition sind diese Maßnahmen als institutioneller Pfadwechsel im konservativ-korporatistischen Wohlfahrtsregime interpretiert worden (Hassel/Schiller 2010). Von nicht minderer Bedeutung war jedoch die Prekarisierung von Arbeit, die durch eine Sequenz von Reformen der Arbeits- und Sozialverfassung ermöglicht und dynamisiert wurde (Castel/Dörre 2009). Zwischen dem schrumpfenden Segment des Normalarbeitsverhältnisses und dem der Arbeitslosigkeit expandiert eine Zone der Prekarität, die Erwerbsformen wie befristete und geringfügige Beschäftigung, Teilzeitbeschäftigung und Leiharbeit, aber auch sogenannte Solo-Selbstständigkeit (Selbstständigkeit ohne Beschäftigung) umfasst. Dabei weist atypische Beschäftigung nicht nur mit Blick auf die institutionelle Integration in die Systeme der Alters- und Arbeitslosenversicherung Defizite auf. Sie geht auch mit einem vierfach höheren Risiko einher, in den mittlerweile auf $22 \%$ angewachsenen Niedriglohnsektor und bereits während der Erwerbsphase in Armutslagen abgedrängt zu werden (Wingerter 2009).

\subsection{VERLUSTE AN ARBEITSMARKT- UND INSTITUTIONELLER MACHT}

Die Folgen des neuen Kommodifizierungsschubes der Arbeitsmarktsubjekte (Promberger 2009) für die gewerkschaftliche Durchsetzungskraft sind dramatisch. So kann die Kombination aus Arbeitslosigkeit und Prekarisierung „vielleicht als die dramatischste Form des Abbaus des Wohlfahrtsstaats" (Korpi/Palme 2003a, S. 236) und zugleich als eine „wesentliche Stärkung der Position der Wirtschaftsinteressen im Verteilungskampf “ begriffen werden (ebd. S. 248).

Einschlägige Studien geben Hinweise auf die Folgen der Prekarisierung von Arbeit für die strukturelle und institutionelle Macht der Gewerkschaften. Es werden weitreichende Einflüsse der Hartz-IV-Reform auf das Verhalten arbeitsuchender Lohnabhängiger und die Strategiebildung betrieblicher Interessenvertretungen konstatiert. So lassen sich über die Altersgruppen hinweg deutliche Tendenzen eines stärker opportunistischen Verhaltens bei Arbeitsuchenden beobachten. Eine Studie des Instituts für Arbeitsmarkt- und Berufsforschung (IAB) stellt fest, dass sich Arbeitsuchende infolge des Hartz-IV-Regimes „häufiger als früher auch um inadäquate Arbeitsplätze bemühen “ und dass befragte Betriebe bekannt gaben, dass „die Konzessionsbereitschaft arbeitsloser Bewerber in Hinblick auf die Lohnhöhe, die Arbeitsbedingungen und das Qualifikationsniveau der Stelle gestiegen" sei (Kettner/Rebien 2009, S. 1).

Doch die Hartz-IV-Reformen veränderten nicht nur das Bewerberverhalten von Arbeitsuchenden, sie werden auch im „Milieu der Insider" des Arbeitsmarktes als massive Bedrohung empfunden (Hassel/ Schiller 2010, S. 98; Herv. i. O). So weisen empirische Befunde darauf hin, dass es insbesondere bei Beschäftigten in Westdeutschland, die das relativ komfortablere Sicherungsniveau der Vor-HartzÄra kannten, „nicht nur unter den real

3 Zur Kritik, die im Rahmen der New-Politics-These an der Klassenorientierung des MachtressourcenAnsatzes formuliert wurde, vgl. Pierson 1996; zur Erwiderung vgl. Korpi/Palme 2003a und 2003b. 
von Arbeitslosigkeit Betroffenen, sondern auch unter Beschäftigten zu einem nachhaltigen Schock durch die so genannten ,Hartz-Reformen' gekommen sein könnte" (Erlinghagen 2010, S. 7; Herv. i. O.). Der erhöhte Administrationsdruck auf Arbeitsuchende und der Hartz-Schock bei Beschäftigten machen auch nicht vor der Praxis betrieblicher Interessenvertretungen halt. So gehen die Analytiker der Bundesbank bei der Erklärung der erheblichen Konzessionen der Betriebsräte gerade in der gegenwärtigen Krisenphase davon aus, dass „,infolge der Sozial- und Arbeitsmarktpolitik in der ersten Hälfte der Dekade die Kompromissbereitschaft der Arbeitnehmervertreter zugenommen hat" (Deutsche Bundesbank 2009, S. 20).

Die Schwächung gewerkschaftlicher struktureller und Organisationsmacht dürfte in den Sektoren der Produktion (Betriebe) und des Tausches (Arbeitsmarkt) auch die Erosion einschlägiger Institutionen im deutschen Arbeitsbeziehungssystem befördert haben. Diese kommt in den betrieblichen Vertretungslücken sowie der rückläufigen Bindung des Flächentarifvertrages zum Ausdruck. Insgesamt ist nur noch eine Minderheit der Beschäftigten in den „Kernzonen des dualen Systems der Interessenvertretung“, also des Geltungsbereichs betriebsrätlicher Interessenvertretungen oder des Tarifvertrages, beschäftigt, was mit negativen Auswirkungen auf die Sicherung gewerkschaftlicher Loyalität verbunden sein dürfte (Pyhel 2007). Dies gilt in besonderem Maße für die Privatwirtschaft. Die weißen Flecken in der Tarif- und Mitbestimmungslandschaft, also Räume ohne legitimierte Interessenvertretung, sind insgesamt beachtlich, wobei der Rückgang des tarif- und mitbestimmungsrechtlichen Schutzes in Ostdeutschland besonders ausgeprägt ist (Ellguth/Kohaut 2010; Bispinck/Schulten 2009).

Ebenfalls bedeutsam für die gewerkschaftliche Durchsetzungsfähigkeit ist die sukzessive Entwertung der Selbstverwaltung in den sozialen Sicherungssystemen. Die Gewerkschaften profitierten lange von der Einbeziehung der Versicherungsnehmer in die Organisation und die Abwicklung der Versicherungen und Leistungen. Das Institut der sozialen Selbstverwaltung bot ihnen direkte Einflusskanäle in einem wichtigen Sozialstaatssektor sowie allgemeine externe Organisationshilfen. Dazu gehörten rechtsverbindliche Normsetzungskompetenz in der Umfallversiche- rung und allgemeine Informationen und Beziehungskapital in den anderen Versicherungszweigen. Obwohl Selbstverwaltungshandeln stets in verbindliche Vorgaben des Staates eingebunden war, boten sich Wege direkter Einflussnahme, die nicht ausschließlich Anweisungen und Kalkülen der Politik unterworfen waren. Nicht ohne Grund galt die Selbstverwaltung lange als „Bastion der demokratisch orientierten Gewerkschaften" (Seils 2009, S. 348).

Auch in diesem Feld bringen institutionelle Transformationen die schwindende Macht der Gewerkschaften zum Ausdruck. Nicht zuletzt sind die Institutionen der sozialen Selbstverwaltung in den Sog marktschaffender Sozialpolitik geraten und gehen mit einer sukzessiven Entmachtung gewerkschaftlicher Interessenvertretungen einher. Insbesondere die grundlegenden Strukturreformen in der Renten-, Arbeitslosen- und Krankenversicherung haben zu einer politisch intendierten „Schwächung der Selbstverwaltungstradition " und einer Ausweitung von Regulierungskompetenzen des Staates beigetragen (Czada 2008, S. 191; vgl. auch Trampusch 2006).

Aus der Regierungsperspektive erwies sich die soziale Selbstverwaltung trotz aller Schwächen oftmals als Hindernis gegenüber politischen Durchgriffsstrategien. Daher zielten Konzepte des Um- und Abbaus der Sicherungssysteme oftmals auch auf eine Entmachtung der Selbstverwaltung oder ihre Indienstnahme im herrschenden Politikinteresse. Diese institutionelle Transformation und regulatorische Entwertung der sozialen Selbstverwaltung ist als Indiz einer politischen Deregulierung auf dem Weg in eine postfordistische Sozialpolitik bewertet worden (Trampusch 2006). Den Gewerkschaften versperrt sie wichtige Einflusskanäle, beendet externe Organisationshilfen und trägt dabei zur Schwächung institutioneller Macht bei.

\subsection{SCHWINDENDE PARTEIPOLITI- SCHE ORGANISATIONSMACHT}

Nach dem Durchbruch der politischen Demokratie standen den Lohnabhängigen mit den sich gründenden Arbeiterparteien Interessenvertretungsorganisationen in den politischen Arenen des Staates zur Verfügung, die zwar die sozio-ökonomische Machtasymmetrie zwischen Kapital und Arbeit nicht aufzuheben vermochten, mit deren Hilfe aber wohlfahrtsstaatliche Politiken implementiert und die Vertei- lungsergebnisse des Marktes respektive der ökonomischen Verteilungskonflikte korrigiert werden konnten. In der international vergleichenden Forschung wird jedoch seit geraumer Zeit in vielen Ländern des entwickelten Kapitalismus ein signifikanter Machtverlust von „Arbeiterparteien“ („center-left-Parties“) und eine wachsende Entfremdung zwischen ihnen und den Gewerkschaften beobachtet. Diese als Entkoppelung der Arbeiterbewegung beschriebe Entwicklung („De-linking of labour“; Piazza 2001) wirkt als Problemverstärker des ohnehin rückläufigen Einflusses der Gewerkschaften in den politischen Arenen.

In Deutschland bündelt sich diese Debatte in der These von der „Erosion gewerkschaftlicher Lobbymacht" (Hassel 2006). Diese geht neben der geschilderten Schwächung korporatistischer Institutionen auch auf personelle Entflechtungen und eine strategische Neuorientierung der „Agenda 2010“-Sozialdemokratie zurück. Auch in Deutschland übernahm die Sozialdemokratie die Rolle eines aktiven Treibers bei der Abwicklung des organisierten Wohlfahrtskapitalismus und der Durchsetzung des liberalisierten Finanzmarktkapitalismus (Cioffi/Höpner 2006). Sie half insbesondere bei der Implementierung eines polit-ökonomischen Regimes, das die Modi der Unternehmensführung und -kontrolle („Corporate Governance“), die materiellen und sozialen Verteilungsmechanismen sowie die Verteilung von Machtressourcen in einer Weise verändert, die gegen die Beschäftigungs- und Verteilungsinteressen der Lohnabhängigen und die Organisationsinteressen der Gewerkschaften gerichtet ist.

Die so entstandenen Spannungen wurden in Deutschland durch „strategische Entflechtungsentscheidungen" (Trampusch 2004, S. 18) auf Seiten der SPD ergänzt, die auf eine generationenvermittelte Entflechtung der Funktionseliten beider Organisationen hinauslief. In dieser neuen Akteurskonstellation nehmen Sozialdemokratie und Gewerkschaften einander mitunter nicht mehr als Ressource (Unterstützer), sondern als Restriktion (Blockierer) bei der Umsetzung ihrer politischen Strategiepräferenzen wahr. Auch wenn erneute krisen-korporatistische Annäherungsversuche vonseiten der Volksparteien unübersehbar sind (Urban 2010a), die Erosion historisch erprobter Kooperationsbeziehungen sowie die strategische Distanzierung einer schwächelnden 
Sozialdemokratie von den Gewerkschaften drängte deren Einfluss in den politischen Arenen zurück.

\subsection{DIE DOWNSIZING-SPIRALE}

Diese kursorische Skizze der Veränderungen in wohlfahrtsstaatlichen Sektoren, die für die Generierung gewerkschaftlicher Macht von hoher Relevanz sind, stützt die eingangs formulierte These einer Korrelation zwischen marktorientiertem Rückund Umbau des Wohlfahrtsstaates und der Erosion gewerkschaftlicher Macht. Offensichtlich waren Wohlfahrtsstaat und Gewerkschaften miteinander in einer Negativ-Entwicklung verbunden, in der die Liberalisierungsschübe der Politik die Schwächung der Gewerkschaften beförderten und in der die machtpolitische Defensive der Gewerkschaften ihrerseits potenzielle Widerstände gegen den Abbau sozialer Rechte verringerte. Während empirische Zweifel an der These eines globalisierungsbedingten „Race-to-bottom-Wettbewerbs" zwischen den unterschiedlichen Wohlfahrtsregimen angemeldet werden (Obinger/Starke 2007), gibt es Hinweise auf eine „Downsizing-Spirale“ im deutschen konservativ-korporatistischen Regime, in der sich der Abbau des Wohlfahrtsstaates und Machtverluste der Gewerkschaften gegenseitig verstärkt haben.

\section{4}

\section{Sozialstaatliche Erneue- rung und gewerkschaft- liche Revitalisierung}

Die wissenschaftlich wie gewerkschaftsstrategisch spannende Frage wäre nun, ob und unter welchen Voraussetzungen sich die Downsizing-Spirale stoppen und die Perspektive einer Positiv-Entwicklung („Upsizing-Spiral“) eröffnen ließe. Die bisherige Argumentation legt nahe, dass eine solche Wende sehr voraussetzungsvoll ist und ohne eine erneuerte Rekommodifizierung von Arbeit und eine Re-Stabilisierung gewerkschaftlicher Organisationsmacht jedoch nicht zu haben sein dürfte. Daher soll im Folgenden - kursorisch und exemplarisch - auf gegenwärtige Reformdebatten und -bemühungen verwiesen werden, die unter den Bedingungen des deutschen Wohlfahrtsregimes die Realisierung dieser Voraussetzung befördern könnten.

\subsection{DE-KOMMODIFIZIERENDE REGULIERUNGEN}

Im Zentrum einer erneuten De-Kommodifizierung von Arbeit müsste die Realisierung eines neuen Arbeitsregimes stehen. Die Integration prekärer (abhängiger wie selbstständiger) Beschäftigung in die sozialen Sicherungssysteme, die Erhöhung von Beschäftigungssicherheit durch einen ausgebauten Kündigungsschutz, die Zurückdrängung sachgrundloser Befristungen sowie die Einschränkung und soziale Regulierung von Leiharbeit („equal treatment") werden als wichtige Elemente in diesem Sinne diskutiert. Ergänzt werden müsste dies durch eine Re-Stabilisierung des Flächentarifvertragsystems. „Bei der Re-Stabilisierung von unten geht es um die Stärkung gewerkschaftlicher Organisationsmacht, um die Tarifbindung auf Branchen- und betrieblicher Ebene verteidigen bzw. Unternehmen wieder neu in die Tarifbindung zwingen zu können. Bei der Re-Stabilisierung von oben geht es darüber hinaus um die politische Stärkung des Tarifvertragssystems. Dabei kommt der Reform der Allgemeinverbindlich-

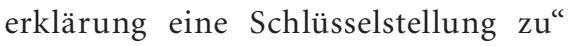
(Bispinck/Schulten 2009, S. 17). Ein weiteres unverzichtbares Element einer dekommodifizierenden Arbeitsmarktverfassung wäre ein flächendeckender Mindestlohn. Dieser hätte eine über Wirtschaftszweige und Sektoren hinwegreichende Untergrenze der Entlohnung sicherzustellen, ohne die vorhandenen Unterschiede mit Blick auf Produktivität, Wettbewerbskraft und Entgelttraditionen außer Acht lassen zu müssen. Dies könnte durch ein Modell realisiert werden, in dem für untere Entgeltgruppen geltende Tarifverträge für allgemeinverbindlich erklärt werden, wobei ein allgemeiner nationaler Mindestlohn die gesetzliche, auch durch Tarifverträge nicht zu unterschreitende Untergrenze darstellen müsste (Schulten 2010).

Individuelle wie kollektive Arbeitsmarktmacht entscheidet sich nicht zuletzt über Regelungen zu Höhe, Anspruchsvoraussetzungen und Sanktionsmaßnahmen bei Arbeitslosigkeit sowie die Vorschriften der Zumutbarkeit mit Blick auf die verpflichtende Arbeitsaufnahme. Unter den Bedingungen des deutschen Arbeitsmarktes liefe dies auf eine grundlegende Korrektur der Hartz-Reformen hinaus. Eckpunkte könnten sein (Urban 2010b):
- die (altersgruppenspezifische) Verlängerung von Bezugszeiten für Arbeitslosengeld (ALG)-I-Bezieher;

- die Anhebung der Regelsätze für ALGII-Bezieher über eine sachgerechte Bedarfsermittlung oder die Wiederankoppelung an vorherige Arbeitseinkommen; - die allgemeine Dynamisierung von Lohnersatz- und Fürsorgeleistungen entsprechend der allgemeinen Einkommensentwicklung;

- Zumutbarkeitsregelungen, die auf administrativen Zwang zur Aufnahme prekärer Arbeit verzichten und Elemente des Berufs-, Qualifizierungs- und Verdienstschutzes enthalten;

- eine Entschärfung der Sanktionsregelungen und die Verbesserung des Rechtsschutzes für die Betroffenen;

- der Verzicht auf die öffentliche Subventionierung von Niedriglöhnen durch die Anhebung von Hinzuverdienstgrenzen.

\subsection{ORGANIZING UND ORGANISATI- ONSMACHT}

Eine dekommodifizierende Arbeitsmarktreform müsste von ergänzenden Anstrengungen der Gewerkschaften zur Erneuerung ihrer Organisationsmacht begleitet werden. Impulse dazu liefert der „Strategic Unionism"-Ansatz in der neueren Gewerkschaftsforschung (Frege/Kelly 2004; Huzzard et al. 2004). Demnach agieren die Gewerkschaften auch in Defensivkonstellationen in einem Möglichkeitsraum mit einer diversifizierten Gelegenheitsstruktur, die ein Spektrum an strategischen Optionen bereit hält, die mit Blick auf die anvisierte Revitalisierung unterschiedlich ergiebig sind (Hyman 2007; Brinkmann et al. 2008).

Insbesondere die Außerwertsetzung (soziale Selbstverwaltung) und die Erosion (Tarifsystem) einst stabiler korporatistischer Aushandlungsinstitutionen machen die Erneuerung eigener gewerkschaftlicher Verhandlungs- und Organisationsmacht notwendig. Dies erfordert eine Umkehr der Mitgliederentwicklung in Richtung eines allgemeinen Mitgliederzuwachses, die Steigerung der Organisationsgrade in den traditionellen Sektoren gewerkschaftlicher Verankerung und schließlich die organisationspolitische Erschließung der neueren Sektoren qualifizierter, weiblicher und junger Dienstleitungsarbeit. In diesem Kontext sind gewerkschaftliche Organizing-Strategien als Maßnahme der 
Wiederherstellung gewerkschaftlicher Organisationsmacht bedeutsam. Bei diesem Beispiel innovativer Praxis handelt es sich um eine offensive Organisierungspolitik, der sich zunächst die angelsächsischen und seit geraumer Zeit auch die deutschen Gewerkschaften zugewandt haben (Dörre 2008; Rehder 2008). Im Kern geht es um die Erweiterung von Mitgliederbasis und betrieblicher Verankerung. Dabei bedient sie sich konfliktorientierter Mobilisierungsund Kampagnenmethoden („Campaigning“), die nicht selten dem Politiktypus von Nicht-Regierungsorganisationen entliehen werden. Zugleich gehen diese Revitalisierungsanstrengungen vielfach mit einer Rückbesinnung auf die politische Ermächtigung der Mitglieder („empowerment“) und einem "Social Movement Unionism“ einher, der eine aktivierte Mitgliederbasis als eigentliches Reservoir gewerkschaftlicher Organisationsmacht anerkennt (Bremme et al. 2007).

\section{Forschungsstrategische Schlussfolgerungen}

Eine Überprüfung der These von der Downsizing-Spirale und die Formulierung prospektiver Vermutungen über das zukünftige Wechselverhältnis von Wohlfahrtsstaat und Gewerkschaftsmacht müsste das Feld von Analysegegenständen und methodischen Perspektiven erheblich erweitern. Nicht zuletzt die Einbeziehung der europäischen Dimension würde Fragestellungen und Analyseperspektiven grundlegend verändern. Doch bereits die eher kursorischen Ausführungen zu den Wechselwirkungen zwischen wohlfahrtsstaatlicher Entwicklung und gewerkschaftlicher Machtausstattung geben Hinweise für die Wohlfahrtsstaats- und Gewerkschaftsforschung.

Dabei kann zum einen an die Debatte über die Schub- und Bremskräfte sozialpolitischer Anstrengungen und die Determinanten gebremster Sozialstaatlichkeit angeknüpft werden (Obinger/Wagschal 2000a). Insbesondere sozio-ökonomische, parteipolitische wie institutionelle Aspekte sind als wichtige Faktoren wohlfahrtsstaatlicher Entwicklung ausgemacht worden, ohne dass eine einzelne der gängigen Theoriefamilien in der Staatstätigkeitsforschung alleinige Erklärungskraft für die wohlfahrtsstaatliche Entwicklungsdynamik beanspruchen könnte. Das spricht dafür, auch den Machtressourcen-Ansatz und mit ihm den Beitrag von Gewerkschaften und arbeitsorientierten Parteien wieder stärker zu seinem Recht kommen zu lassen. Das gilt für die Überprüfung der „New Politics“-These in der deutschen Entwicklung wie für die Forschung über die allgemeinen machtpolitischen Voraussetzungen wohlfahrtsstaatlicher Erneuerung.

Auch in vielen Arbeiten der „Labor Revitalization Studies“ kommt zu kurz, was der kombinierte Machtressourcen-Ansatz als Schlussfolgerung nahelegt: Dass unter den Bedingungen einer voranschreitenden Kommodifizierung von Arbeit und der Liberalisierung sozialstaatlicher Regulierungen den Gewerkschaften eine Revitalisierung aus eigener Kraft kaum gelingen dürfte. Wenn diese Einschätzung berechtigt ist, so kann dies weder für die gegenwärtige Gewerkschaftsforschung noch für aktuelle gewerkschaftliche Strategiedebatten konsequenzlos sein. Überfällig scheint eine systematische Forschung über die Voraussetzungen der Regeneration gewerkschaftlicher Machtressourcen und die machtpolitischen Vorrausetzungen der Realisierung vorhandener „strategischer Optionen“ in der gegenwärtigen Kapitalismusformation. Vor allem die Entstehung und Anwendung von kommunikativer Gewerkschaftsmacht in modernen „MedienGesellschaften" stellt ein wissenschaftlich bisher kaum bearbeitetes Feld dar. Insgesamt liefe dies auf die überfällige Arbeit an einer Theorie gewerkschaftlicher Macht im Finanzmarkt-Kapitalismus hinaus. Anschlussfähig wäre dies an die aktuelle Revitalisierungs-Forschung, aber auch etwa an die Forschungen zur Bedeutung von „klassenübergreifenden Reformkoalitionen " für sozialstaatliche Pfadwechsel (z. B. Hassel/Schiller 2010, S. 98ff.) und die Frage, welche Rolle Gewerkschaften in diesen spielen könnten (Hyman/GumbrellMcCormick 2010b).

\section{Gewerkschaftsstrategischer Ausblick}

Auch die gewerkschaftliche Strategiedebatte könnte von dieser Forschungsperspektive profitieren. Wenn mit der De-Kommodifizierung der Arbeit und der Präsenz der Gewerkschaften in marktregulierenden Institutionen ihre strukturelle und Organisationsmacht steigt, dann spricht dies entschieden gegen eine gewerkschaftliche Strategieorientierung, die diese wohlfahrtsstaatlichen Sektoren ausklammert und sich auf das vermeintliche Kerngeschäft der Betriebs-, Tarif- und Organisationspolitik zurückzieht. So wichtig die Erneuerung von Macht und Institutionen in diesen Sektoren ist: Ohne einen Stopp der Downsizing-Spirale und Erfolge bei deren Umkehr dürften umfassende organisationspolitische Revitalisierungsbemühungen zum Scheitern verurteilt sein. Dazu ist die Reformulierung eines offensiven sozialpolitischen Mandats der Gewerkschaften keine hinreichende, aber sicherlich eine unabdingbare Vorrausetzung (Urban 2005). Diese hätte sich sodann in Strategien der Bekämpfung von Arbeitslosigkeit und Prekarität sowie in Reforminitiativen in der Altersicherungs- und allgemeinen Sozialstaatspolitik zu konkretisieren.

Für die Gewerkschaften dürften sich die Kapitalismus-Krise und die absehbaren liberalisierungs- und austeritätsorientierten Krisenstrategien der Staaten zu elementaren Bedrohungen auswachsen. Bisher scheinen sie nicht in der Lage, aus der offensichtlichen Diskreditierung neoliberaler Politiken Kraft und Kapazitäten für Gegenwehrstrategien zu generieren (dazu etwa Baccaro 2010); zu umfassend war offenbar der Prozess der Erosion gewerkschaftlicher Macht in allen seinen Dimensionen, als dass diese unter den erschwerten Bedingungen der Krise zu strategischen Interventionen im Sinne einer proaktiven Einflussnahme auf die dominierenden Krisenstrategien in der Lage wären. Mit Bereitschaft zu Konflikt und Militanz allein ist es nicht getan, wie die Entwicklungen in unterschiedlichen Ländern deutlich werden lassen. Ohne die notwendigen Machtressourcen erscheinen sie mitunter eher als Ausdruck strategischer Ratlosigkeit. Nicht selten korrespondieren radikale Aktionsformen mit eher defensiven Zielen, während kompromissorientiertere Strategien sozialer Dialoge und Pakte weiterreichende Erfolge zu realisieren scheinen (Hyman/GumbrellMcCormick 2010a).

Dies könnte auch für Deutschland der Fall sein. Dort haben die Gewerkschaften mit Unternehmensvorständen und staatlicher Arbeitsverwaltung einen signifikanten Beitrag zur Sicherung von Be- 
schäftigung und zum „deutschen Arbeitsmarktwunder" erbracht (Möller 2010). Während in anderen Ländern Europas eine Radikalisierung betrieblicher und gewerkschaftlicher Gegenwehr (etwa in Form von „Bossnapping“ oder politischen Generalstreiks, vgl. hierzu Artus in diesem Heft) $\mathrm{zu}$ beobachten war, haben die deutschen Gewerkschaften auf einen betrieblichen und politischen Krisen-Korporatismus gesetzt, der von der Politik erwidert und mit der Erwartung deeskalierender Krisenhilfe verbunden wurde (Urban 2010a). Doch auch hier werden die gewerkschaftlichen Bäume nicht in den Himmel wachsen. Ohne die Erneuerung autonomer gewerkschaftlicher Macht und hinreichende Konfliktfähigkeit wird diese Politikform für die Gewerkschaften riskant bleiben. Sie dürfte in dem Maße an Grenzen stoßen, in dem Interessenkollisionen zwischen Staat, Kapital und Arbeit und entsprechende Verteilungs- und Aushandlungskonflikte zunehmen. Und diese werden im Zuge der Krisenkonsolidierungsbemühungen nicht lange auf sich warten lassen. Eine alternative Strategie der autonomen, machtbasierten Interessenorientierung wäre der krisenkorporatistischen Einbindung allerdings nur dann überlegen, wenn die erreichbaren Verteilungs- und Organisationserfolge den Gehalt der interessenpolitischen Zugeständnisse übersteigen, die im politischen Tausch zwischen Gewerkschaften, Kapital und Staat im krisenkorporatistischen Arrangement realisierbar sind - und diese waren, etwa in Form beschäftigungspolitischer Schutzwälle gegen die externen Schockwellen der Finanzmarktkrise, durchaus ansehnlich.
Der machtressourcen-theoretische Blick lässt deutlich werden: Interessenpolitische Erfolge der Gewerkschaften sind ein machtpolitisch äußerst anspruchsvolles Unterfangen. Sie sind nicht nur eine Frage des strategischen Wollens, sondern zugleich des machtpolitischen Könnens und damit der hinreichenden Ausstattung mit Machtressourcen - sei es in Form von struktureller, institutioneller, kommunikativer oder Organisationsmacht. Dies gilt für konfliktorientierte und militantere wie für kompromiss- und dialogorientierte Strategien. Somit wird die Regeneration gewerkschaftlicher Macht im Finanzmarkt-Kapitalismus zur generellen Bedingung erfolgreicher Interessenpolitik, gleichgültig, ob „boxing or dancing“ (Huzzard et al. 2004) die passende Metapher dazu liefert.

\section{LITERATUR}

Baccaro, L. (2010): Does the global finance crisis mark a turning point for labour?, in: Socio-Economic Review 2, S. 341-348

Bispinck, R./Schulten, T. (2009): Re-Stabilisierung des deutschen Flächentarifvertragssystems, in: WSI-Mitteilungen 4, S. 201-209

Boyer, R. (2000): Is a Finance Led Growth Regime a Viable Alternativ to Fordism?, in: Economy \& Society 1, S. 111-145

Bremme, P./Fürniß, U./Meinecke, U. (Hrsg.) (2007): Never work alone. Organizing - ein Zukunftsmodell der Gewerkschaften, Hamburg Brinkmann, U./Choi, H./Detje, R./Dörre, K./Holst, H./Karakayali, S./ Schmalstieg, C. (2008): Strategic Unionism: Aus der Krise zur Erneuerung? Umrisse eines Forschungsprogramms, Wiesbaden

Castel, R./Dörre, K. (Hrsg.) (2009): Prekarität, Abstieg, Ausgrenzung. Die soziale Frage am Beginn des 21. Jahrhunderts, Frankfurt/New York Cioffi, J.W./Höpner, M. (2006): Das parteipolitische Paradox des Finanzkapitalismus. Aktionärsorientierte Reformen in Deutschland, Frankreich, Italien und den USA, in: Politische Vierteljahresschrift 3, S. $419-440$

Czada, R. (2008): Irrwege und Umwege in die neue Wohlfahrtswelt, in: Heinze, R./Evers, A.(Hrsg.): Sozialpolitik. Ökonomisierung und Entgrenzung, Wiesbaden, S. 186-207

Deutsche Bundesbank (2009): Monatsbericht Dezember 2009, Frankfurt/M.

Dörre, K. (2008): Die strategische Wahl der Gewerkschaften. Erneuerung durch Organizing?, in: WSI-Mitteilungen 1, S. 3-10

Dörre, K. (2010): Überbetriebliche Regulierung von Arbeitsbeziehungen, in: Böhle, F./Voß, G./Wachtler, G. (Hrsg.): Handbuch Arbeitssoziologie, Wiesbaden, S. 873-912

Ellguth, P./Kohaut, S. (2010): Tarifbindung und betriebliche Interessenvertretung: Aktuelle Ergebnisse aus dem IAB-Betriebspanel 2009,

in: WSI-Mitteilungen 4, S. 204-209
Erlinghagen, M. (2010): Mehr Angst vor Arbeitsplatzverlust seit Hartz? Langfristige Entwicklung der Beschäftigungsunsicherheit in Deutschland, IAQ-Report 2

Esping-Andersen, G. (1985): Politics against Markets. The Social Democratic Road to Power, Princeton

Frege, C./Kelly, J. (Hrsg.) (2004): Varieties of Unionism: Strategies for Union Revitalisation in an Globalizing Economy, Oxford

Haug, W. F. (2009): Gewerkschaften im High-Tech-Kapitalismus vor der Hegemoniefrage, in: Das Argument 6, S. 879-893

Hassel, A. (2006): Erosion gewerkschaftlicher Lobbymacht, in: Leif, T./ Spehr, R. (Hrsg.) (2006): Die fünfte Gewalt. Lobbyismus in Deutschland, Wiesbaden, S. 188-198

Hassel, A./Schiller, C. (2010): Sozialpolitik im Finanzförderalismus. Harz IV als Antwort auf die Krise der Kommunalfinanzen, in: Politische Vierteljahresschrift 1, S. 95-117

Höpner, M. (2009): „Spielarten des Kapitalismus“ als Schule der vergleichenden Staatstätigkeitsforschung, in: Zeitschrift für vergleichende Politikwissenschaft 3, S. 303-327

Höpner, M./Petring, A./Seikel, D./Werner, B. (2009): Liberalisierungspolitik. Eine Bestandsaufnahme von zweieinhalb Dekaden marktschaffender Politik in entwickelten Industrieländern, Max-Planck-Institut für Gesellschaftsforschung (MPIfG), Discussion Paper 7, Köln

Huzzard, T./Gregory, D./Scott, R. (Hrsg.) (2004): Strategic Unionism and Partnership. Boxing or Dancing?, New York

Hyman, R. (2007): How can trade Unions act strategically?,

in: Transfer 2, S. 193-210

Hyman, R./Gumbrell-McCormick, R. (2010a): Trade unions and the crisis: a lost opportunity?, in: Socio-Economic Review 2, S. 364-372

Hyman, R./Gumbrell-McCormick, R. (2010b): Trade Unions, Politics and Parties: Is a New Configuration Possible? Unveröffentlichtes Manuskript 
Kettner, A./Rebien, M. (2007): Hartz IV-Reform. Impulse für den Arbeitsmarkt, IAB-Kurzbericht 19, Nürnberg

Korpi, W. (1983): The Democratic Class Struggle, London

Korpi, W. (1998): The Iceberg of Power below the Surface: A Preface to Power Resources Theory, in: O'Connor/Olson (Hrsg.): Power resources theory and the welfare state, Toronto, S. vii-xiv

Korpi, W./Palme, J. (2003a): Klassenpolitik und Wohlfahrtsstaatsabbau: Kürzungen von Rechten der sozialen Sicherung in 18 Ländern 1975-1995, in: Müller, W./Scherer, S.(Hrsg.): Mehr Risiken - Mehr Ungleichheit?, Frankfurt/New York, S. 221-255

Korpi, W./Palme, J. (2003b): New Politics and Class Politics in the Context of Austerity and Globalization: Welfare State Regress in 18 Countries, 1975-95, in: American Political Science Review 3, S. 425-446

Lütz, S./Czada, R. (Hrsg.) (2004): Wohlfahrtsstaaten. Transformation und Perspektiven, Wiebaden

Möller, J. (2010): The German labor market response in the world recession - de-mystifying a miracle, in: Zeitschrift für Arbeitmarktforschung 4, S. 325-336

Obinger, H./Starke, P. (2007): Sozialpolitische Entwicklungstrends in OECD-Ländern 1980-2001: Konvergenz, Divergenz oder Persistenz, in: Holzinger, K./Jörgens, H./Knill, C. (Hrsg.): Transfer, Diffusion und Konvergenz von Politiken, PVS Sonderheft 38, Wiesbaden, S. 470-495 Obinger, H./Wagschal, U. (2000a) (Hrsg.): Der gezügelte Wohlfahrtsstaat. Sozialpolitik in reichen Industrienationen, Frankfurt/M.

Obinger, H./Wagschal, U. (2000b): Ökonomie, Institutionen und Politik: Determinanten gebremster Sozialstaatlichkeit im Überblick, in: Dies. (Hrsg.) (2000): Der gezügelte Wohlfahrtsstaat. Sozialpolitik in reichen Industrienationen, Frankfurt/M., S. 365-388

O'Connor, J./Olson, G. (Hrsg.)(1998): Power Ressources Theory and the Welfare State. A critical Approach, Toronto

Piazza, J. (2001): De-Linked Labor. Labor Unions and Social Democratic Parties under Globalisation, in: Party Policies 4, S. 413-435

Pierson, P. (1996): The new Politics of the Welfare State, in: World Politics 1, S. 143-179

Pierson, P. (Hrsg.) (2001): The New Politics of the Welfare State, Oxford
Promberger, M. (2009): Fünf Jahre SGB II. Versuch einer Bilanz, in: WSI-Mitteilungen 11, S. 604-611

Pyhel, J. (2007): Betriebliche Mitbestimmung und gewerkschaftliche Loyalitätssicherung, in: Industrielle Beziehungen 1, S. 32-52

Rehder, B. (2008): Revitalisierung der Gewerkschaften?, in: Berliner Journal für Soziologie 3, S. 432-456

Sachverständigenrat zur Begutachtung der gesamtwirtschaftlichen Entwicklung (SVR) (2008): Die Finanzkrise meistern - Wachstumskräfte stärken, Gutachten 2008/2009 (o.O.)

Schulten, T. (2010): WSI-Mindestlohnbericht 2010. Unterschiedliche Strategien in der Krise, in: WSI-Mitteilungen 3, S. 152-159

Seils, E. (2009): Die Sozialversicherung im internationalen Vergleich, in: WSI-Mitteilungen 7, S. 347-354

Streeck, W. (2009): Von der gesteuerten Demokratie zum selbststeuernden Kapitalismus. Die Sozialwissenschaften in der Liberalisierung, in: WestEnd 1, S. 13-33

Trampusch, C. (2004): Von Verbänden zu Parteien. Der Elitenwechsel in der Sozialpolitik, Max-Planck-Institut für Gesellschaftsforschung (MPIfG), Discussion Paper 3, Köln

Trampusch, C. (2006): Postkorporatismus in der Sozialpolitik - Folgen für Gewerkschaften, in: WSI-Mitteilungen 6, S. 347-352

Urban, H.-J. (2005): Gewerkschaften als konstruktive Vetospieler, in: Forschungsjournal Neue Soziale Bewegungen 2, S. 44-60 Urban, H.- J. (2010a): Niedergang oder Comeback der Gewerkschaften, in: Aus Politik und Zeitgeschichte 13-14, S. 3-7

Urban, H. J. (2010b): Für einen arbeitsmarktpolitischen Neustart. Sicherheit und Perspektiven statt Hartz IV, in: Soziale Sicherheit 2, S. $45-52$

Windolf, P. (Hrsg.) (2005): Finanzmarktkapitalismus. Analysen zum Wandel von Produktionsregimen, Wiesbaden

Wingerter, C. (2009): Der Wandel der Erwerbsformen und seine Bedeutung für die Einkommenssituation Erwerbstätiger, in: Wirtschaft und Statistik 11, S. 1080-1098

Wright, E.O. (2000): Working-Class Power, Capitalist-Class Interests, and Class Compromise, in: American Journal of Sociology 4, S. 957-1002 